(C) EDP Sciences, 2009

DOI: $10.1051 /$ orvie/2009005

\title{
In the beginning there was shape
}

\author{
S. Douady, A. Manning and B. Hennion
}

\begin{abstract}
The gist of the ideas presented here was formulated while trying to bridge the gap between a simple physical conception of the world and the global philosophy of life of A. Manning. Some ideas about Energy Consumption and Coding were further developed in a group discussion with Sophie Heinrich, Maël Montevil, Arnaud Pocheville and Livio Riboli-Sasco, whom it is our pleasure to thank here.
\end{abstract}

\section{SHAPE IS THE ESSENCE OF LIVING SYSTEMS}

When Biology first appeared, long before its recent mutation into Molecular Biology, it was occupied with the description of the shapes of the different living beings. Shapes are difficult to describe. They are most easily transmitted by drawings, on which names can be ascribed, helping to outline the elements of simple shapes. Similar elements can be recognized in different living life forms and used to group them, with both their similarities (same elements) and differences (element shaped slightly different). Thus, it is easy to make abstract categories such as leaf, even though leaves differs greatly in the details of their shapes, and group similar individuals (organisms) by species, family, genus, etc.

The fact that these distinctions are meaningful comes from the information that shapes reveal a deep organization of living beings (usually associated with functions) that are shared throughout the living world.

Biology eventually became focused on the transmission of shapes through generations (genetics) and the explanation of the similarities of shapes by a slow variation (evolution). In so doing, the tendency is to forget the shapes as entities, in and of themselves.

\section{THE DIFFERENCE BETWEEN LIVING AND INERT OBJECTS}

Inert object also have shapes. Crystals can be recognized by their characteristic shapes and colours. As first notice by Botanist L. Bravais, who became a crystallographer, shapes reveal internal organization at an atomic level.

The main difference lies, not in the existence of characteristic shapes, but in the dynamics. Inert objects are, by definition, inert and do not change in time. If they do change, it is only by the aggression of being chemically and mechanically attacked or melted by the outside environment. Because crystals have a passive system of defence the pieces that comprise them remain unchanged over time. As a result some crystals can give us the age of the earths formation.

Similarly, living systems succeed in keeping their shape, but they do so in a completely different way. They preserve their organization by constantly demolishing and recreating, as the elements are changing regularly. For instance, blood cells are changed weekly

Article available at http://www.origins-and-evolution.org or http://dx.doi.org/10.1051/orvie/2009005 


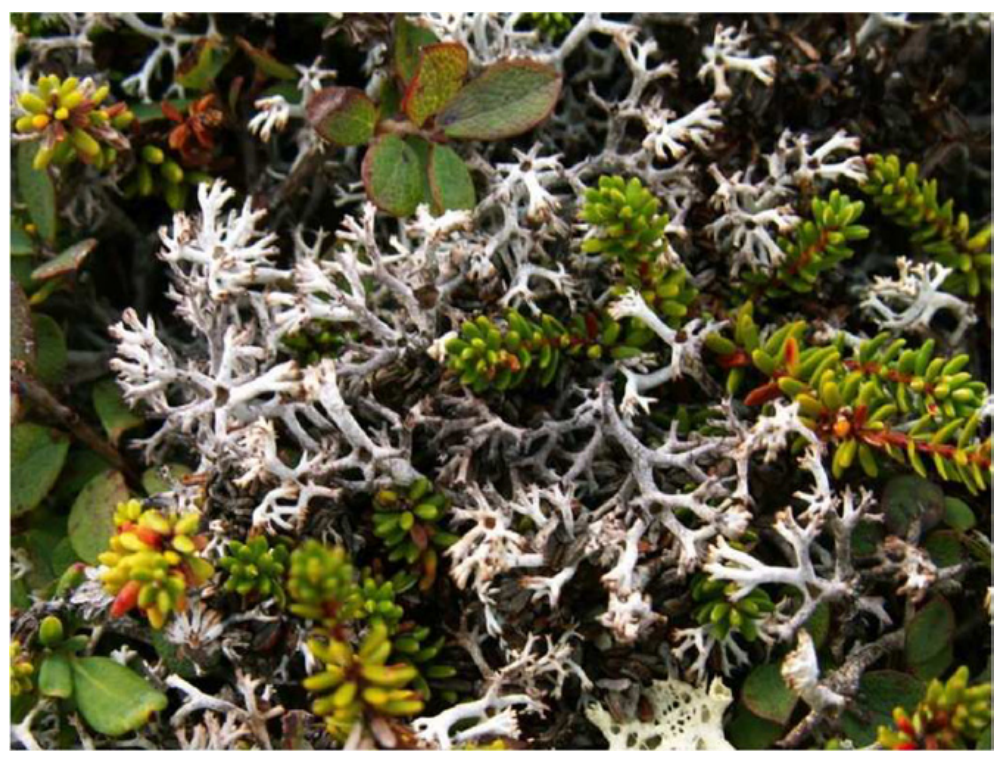

Figure 1. At first glance, in this picture, one can recognize different species of plants even without knowing them beforehand. This is because they have elements that we already know and are able to recognize (leaves), with distinctly different shapes and colours. From previous knowledge, we attribute these differences to a difference in species.

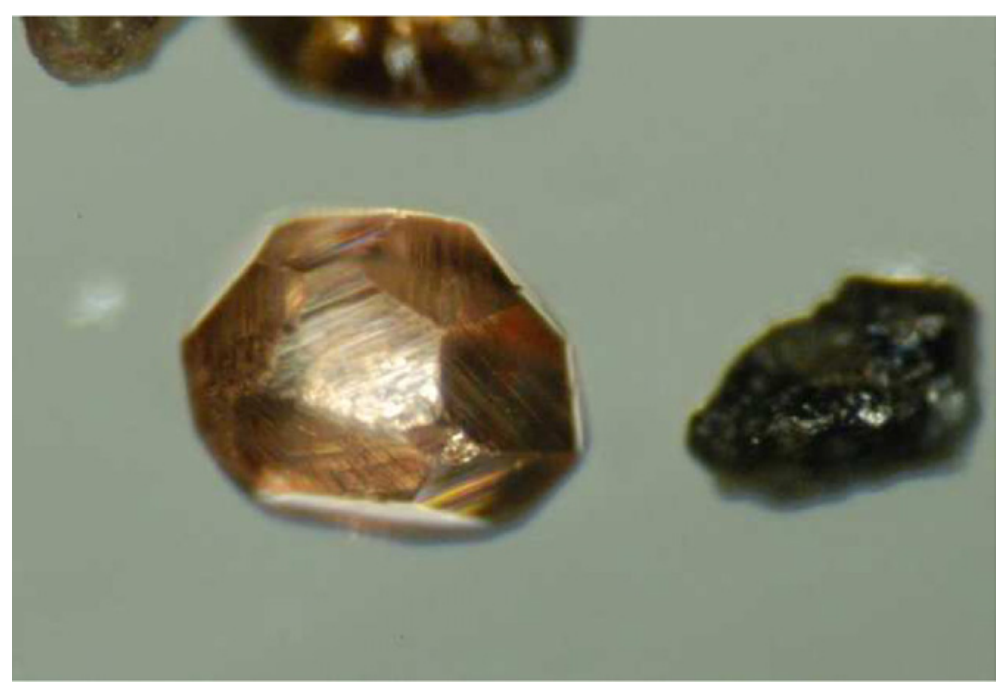

Figure 2. A crystal. Its particular shape reveals the symmetry of the underlying structure at the atomic scale. But once put in place, the element of which it is comprised will not move, until erosion eventually reaches them. 
and bones are dissolved and synthesized constantly. Our cells are dying and regenerating relentlessly. In essence, the shape of a living organism is maintained by constant activity. Constant change creates short-term problems, as the organism has to cope with ongoing regeneration.

The following example illustrates the different approach. In a garden in Kyoto a small building, the Golden Pavilion, has stood for hundreds of years. Western tourists come to admire this architecture from ancient times. However, they are shocked when they realize that in fact, through permanent repairs, there does not remain any part of the structure that existed when the Golden Pavilion was first constructed. Not only the gold cover but every piece of wood has been changed. For Westerners, the building is no longer the real Golden Pavilion but merely a recent reproduction. On the contrary, Asian tourists cannot understand this point of view. For them it is clearly the real Golden Pavilion, which they have succeeded in preserving in its original pristine state. This demonstrates that Westerners consider buildings as dead, Inert Objects, while Asians consider built architecture as Living Organisms, which require permanent change to remain identical.
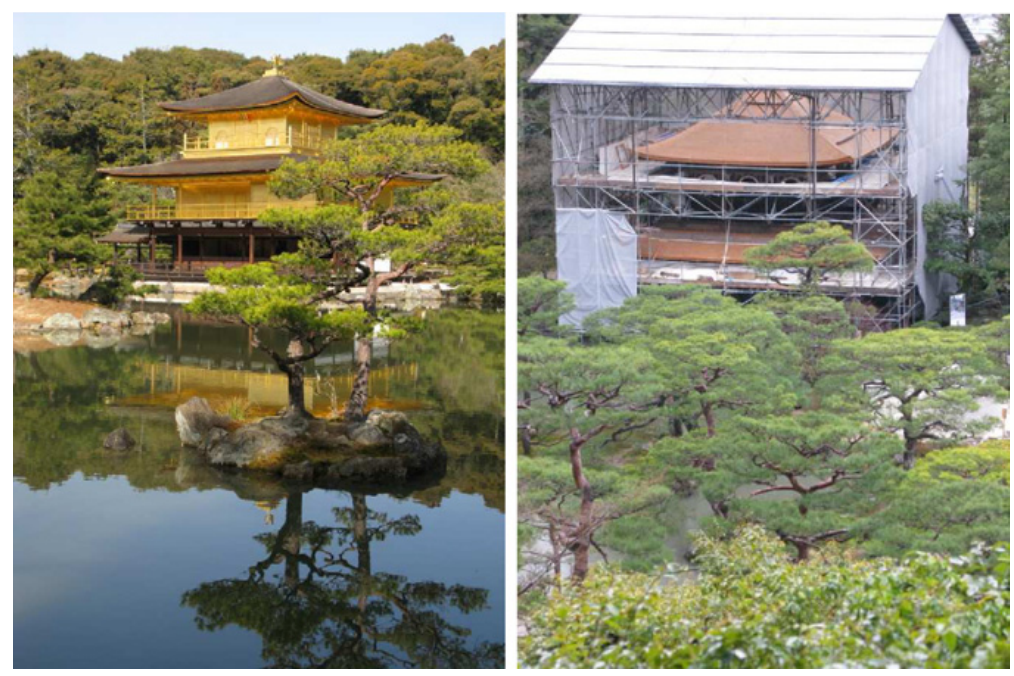

Figure 3. The Golden and Silver Pavilions in Kyoto (golden, right, silver, in repair, left). Through their numerous repairs, are they still the same Pavilions, or have they become reproductions? Are they Inert Object, aging slowly and eventually disappearing, or Living Organism, staying the same and alive by permanent repairs?

\section{SHAPE CAN MAINTAIN ITSELF ONLY BY VIRTUE OF ITS OWN ORGANIZATION}

With the discovery of the molecular support for the genetic transfer of shape though generations, and even more so the technical possibility of playing with it, Biology became focused on its code-like inscription to explain every aspect of life. The presence of DNA or 
RNA even became the definition of life. However, one can argue that there were structures, able to maintain themselves, prior to the appearance of genetic coding.

First it is hard to imagine that coding appeared for something that did not exist, and then created it. This resembles Intelligent Design. There are simple enclosed chemicals systems that were built that are able to maintain themselves (until the exhaustion of the environment) without the intervention of a coded orchestra chief.

A purely physical example, that of sand dunes, can give an understanding of how shape, by virtue of its own existence, can regulate and maintain itself, without any other intervention. Barchan dunes are isolated dunes that form with a small amount of sand when the wind keeps the same direction constantly.
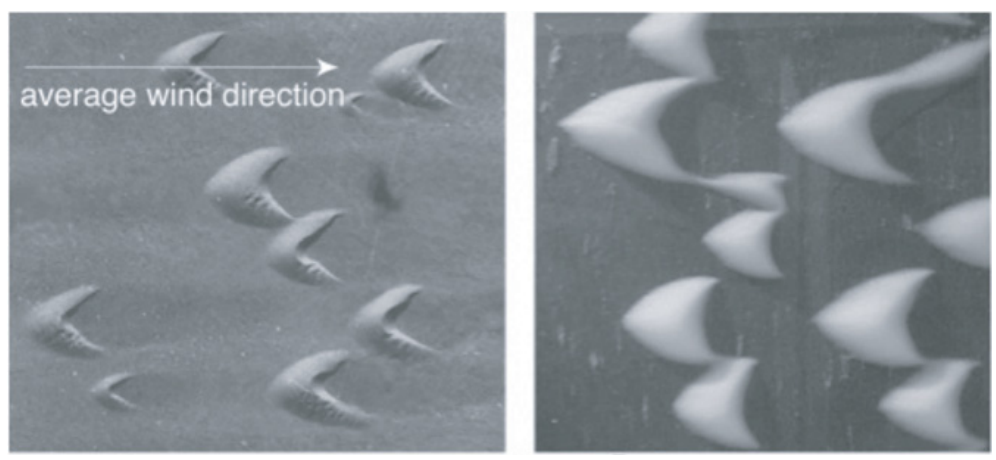

Figure 4. (left) An aerial view of Barchans in the Moroccan Desert (width approximately $100 \mathrm{~m}$ ), and (right) a smaller scale lab equivalent.

Barchan dunes are crescent dunes that propagate with their two horns downwind. The particularity of these dune is to move down the wind as a whole, preserving perfectly their shape. There is no coded information book of instructions to tell the dunes how its shape should be, either in the wind or in the sand grains. So where does this shape and its maintenance comes form?

Dunes are formed and advance with the wind taking up the grains, and dropping them after they form themselves into a crest. Smaller dunes move faster as there is less sand to move. More precisely the sand dunes moves with speed inversely proportional to their height. This reasoning can be applied to separate parts of a dune, and this is why starting from a sand pile, the two sides that are smaller will move faster and end up creating two horns downwind. This is the simple origin of the crescent shape.

The question is: why doesnt this shape deform continuously, with the horns going further away from the center? Why does it moves globally as a whole? This is because the sand, pushed by the wind, also feels the lateral slope, and follows it. Then, the sand flux moves away from the center and rolls toward the two side horns. The wind can take only a limited amount of sand. Receiving the sand from the center, the side wind stops erosion of the horns. Stopping the erosion stops the horns from moving.

There is a spontaneous equilibrium that appears; the sides being thinner move ahead more quickly and create horns. Those horns by being ahead induce lateral sand fluxes that 


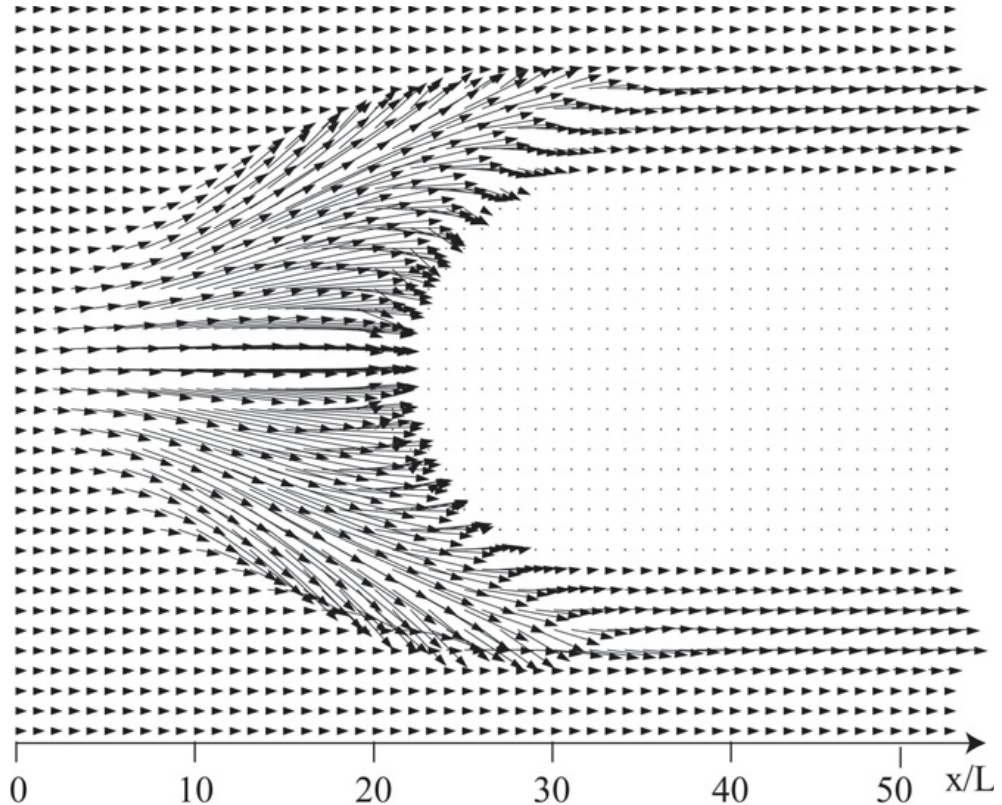

Figure 5. The drawing of the sand flux on the top of a barchan dune. It is the deviation of this flux on the side on the two arms, due to the lateral slope, that slows them and allows the barchan to move as a whole.

slow them down. Eventually the position of the horns adapts so that both the horns and the center move globally at the same speed.

The dune shape is the result of the sand flux but the sand flux (and its essential lateral part) is precisely directed by the dune shape. So barchan dunes are able to maintain their shape through direct self-regulation; the shape directs the flux of sand that keeps the shape unchanged. In this way the shape of the barchans is able to maintain itself. Therefore barchan dunes give an example of a well defined physical structure, which maintains itself without any external or internal source of regulation, but only through the virtue of its own existence.

\section{SPATIAL INSCRIPTION}

Shape is the translation of spatial structure and organization. The sand dune, an organized inscription in space, is usually seen as a passive result from all the other processes... the fluxes of mater and energy. However, the dune also gives the understanding that this spatial inscription is itself a leading element that directs the essential fluxes. To consider the shape, rather than the fluxes, as the leader, is a way to turn the tables, and change the order of the way things are perceived.

In observing the existence of fluxes, the metabolisms which exist in living organisms, and not understanding how such a complex organization came to be, barchan dunes make it easy to postulate that shape was the beginning of the process. The shapes observed 
do not only maintain complex organizations but generate and maintain the fluxes that maintain the shapes.

In living beings, such as biological cells, the main maintenance operations are achieved through fluxes that control chemical reactions. Chemical reactions happen when two molecules come close enough to induce an energetic reaction that exchange atoms and ions, transforming them. In this way cells are using a latent chemical energy and we see that the key parameter is the spatial position of the molecules. Therefore, it is not surprising that the basic feature of cells is to be composed of spatial structures on the scale of chemical interactions. Again, such structures are not only the result of chemical interactions, but, in fact, the spatial shapes that are going to control the spatial position of the molecules in order to control the chemical reactions.

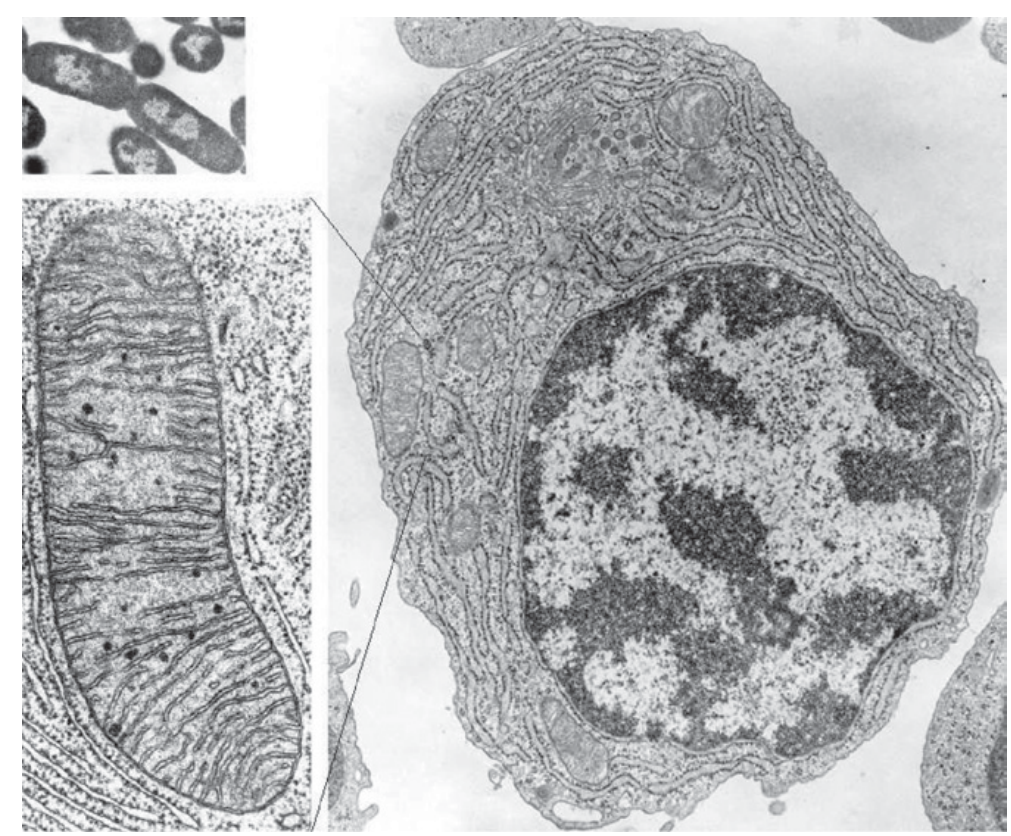

Figure 6. A small prokaryote (the bacteria Escherichia Coli), with only the irregular unclosed nucleus visible, and an Eukaryote cell (roughly at the same scale), with its enclosed nucleus, Endoplasmic Reticulum decorated with ribosome, Mitochondria (enlarged) and Golgi Complex visible.

This is exemplified in Eukaryotes known to possess many compartments folded on this chemical length interaction. But even prokaryotes, that do not possess these compartments, show spatial organization, with fibbers and nests, vesicles and motors driving them along the fibbers, etc... Eukaryotes are much larger than Prokaryotes, which means that more spatial structure, in the form of compartments, were necessary to keep the control of the spatial interactions when increasing the size. In this way it is hard to imagine that the content of the cells could have first been thought of as an unstructured gel, the cytoplasm, like a chemical mixture, where random reactions would occur. 
It is also difficult to imagine that such complex structures as the rough Endoplasmic Reticulum, the Mitochondria and the Golgi Complex are shaped by some controlling architect. It is much easier to think that they structure and maintain their shapes through self-interactions and regulated instabilities, and that these structures directly induce and control the fluxes that maintain them through permanent renovation.

This is not so different from Inert Objects. For instance in the growth of a crystal, there is no instruction book to tell new elements where to go. New elements are guided by the spatial interactions with the already fixed elements and settle accordingly. The only difference is that this step by step spatial organization, instead of leading toward the growth of an inert object, is permanently repeated to maintain Living Organisms.

If we take the usual genome oriented point of view, it is difficult to imagine how coded information can control the spatiality of the cell, and direct all the necessary elements at their respective positions. How could it be informed of the spatial distribution of the elements, and respond to it spatially? It is clear that it can do so only through an existing spatial organization. Even more, the code is spatially distributed along a long linear molecule, and its spatial state, more or less enrolled in chromatin, controls its expression. Even the spatial position, close to a nucleus pore or buried in the center. In this way the mental hierarchy that is underlying simple descriptions, stating that the genome controls what happens in the cell, could be reversed. It can be stated that the spatial cell structure, in the egg cell for instance, controls its own self-maintenance and development, using the information spatially encrypted in the genome.

This discussion on the spatial extension is an essential point for all the spatial extensions of life, i.e. all the shapes: Only an already spatially existing shape can control and direct other spatial extensions.

\section{THE UNNECESSARY INVENTION OF A CODE?}

Following this interpretation, we can see that the presence of a genetic code is not essential compared to the presence of an already physical and spatial organization. If we want to seek for the origin of life, it is reasonable to think that this type of organization appeared and was present before a genetic code appeared.

The genetic code and its support molecules are pervasive throughout living systems, and the dynamics of its transcription prove to be essential daily. So we could ask why such an overwhelming presence now, when it seems clear that it was not present in the beginning.

A possible interpretation is that self-sustaining shapes had to survive in ever changing environments. To be able to survive they had to develop and maintain energy circuits adapted for each new situation. But, as these regulations are necessarily spatially inscribed, they would have used a large physical space. Maintaining spatial structures takes unnecessary energy, especially if their use is not permanent but only by fluke. Coding could then be interpreted as an efficient way to compile, in a recipe book, all these regulation and energy consumption mechanisms, while getting rid of them in the physical space. If needed, the recipe could be read and the necessary structure resynthesized. In this interpretation coding appeared as a useful compaction to reduce the spatial inscription, while potentiality keeping the functionality. 


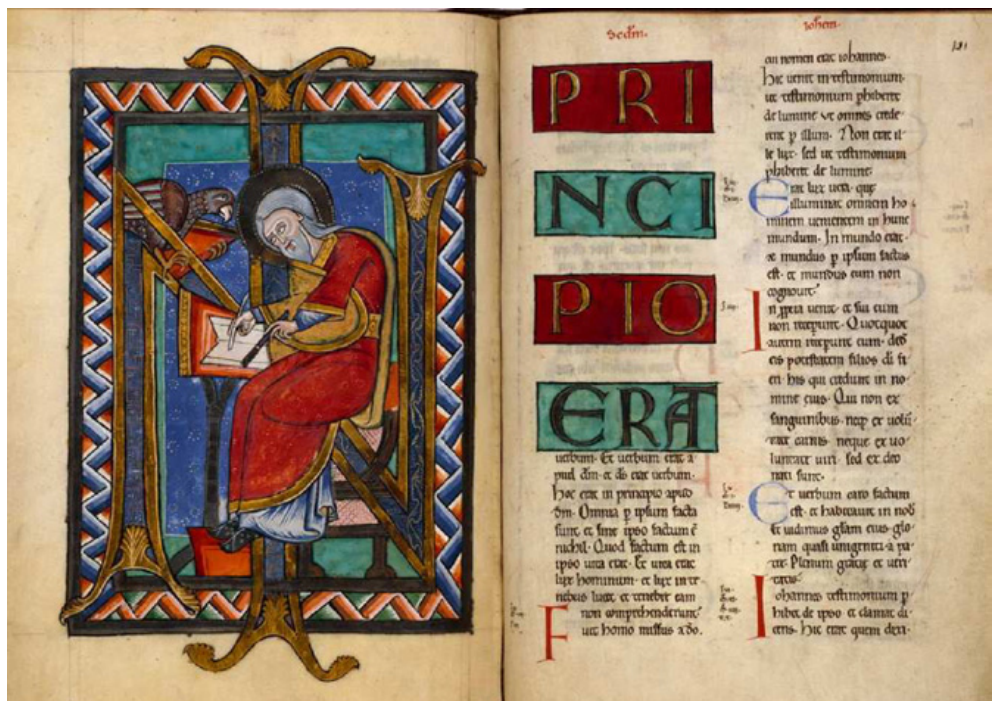

Figure 7. First page of St Johns Gospel. The firsts large letter read In Principio Erat which means In the Beginning Was (the Word-DNA/RNA-Shapes...).

Inventing a code seems to have been a useful invention, as it invaded life. However, we can see that it has its drawbacks. Relying on a book of recipes to know how to react is also a way to lose one's freedom, as the reactions one can have are then directed and stereotyped. This explains the surprising stability of shapes. Consequently, the main problem of life is to escape, one way or another, from this dangerously fixed behaviour. In this way, mutations, sex and evolution can simply be seen a desperate attempt to escape the unfortunate consequence of the invention of code compaction.

\section{LIVING IS EATING}

If we go away from this code, we can come back to the more fundamental consequences of living organisms having to maintain themselves. Permanent change cannot be achieved without consumption from the outside. Even if the elements are reused and their loss is reduced as much as possible, reuse requires energy, which needs to be absorbed from outside. Thus a living organism can maintain its structure only by constant absorption of materials and energy, and the rejection of waste. We know that we cannot survive without breathing for more than few minutes, drinking for more than few days, and eating for more than few weeks. We have a tendency to forget the equivalent amount of waste we reject. Even plants need light, gases, water and minerals, and expel waste.

The constant internal movement and regulation to keep everything unchanged is the focus, in Biology, of the study of Metabolism. Stating that parameters remain constant in living organism can be seen as a deep mystery, or it can just be seen as a simple consequence of preserving the organization itself. 
Being a living organism means existing at the expense of the environment. So contrary to Inert Objects that resist passively the aggression of their environment, Living Organisms are actively marauding their environment.

\section{AVAILABILITY OF ENERGY...}

This living strategy to reshape permanently is energetically demanding, as it needs a constant flux of energy. This is the opposite of Inert Objects, which just need energy for their formation and then avoid it, as any further influx is destructive for them. The environment suitable for life can be conceived only if there is a generous constant source of energy. That happen to be conveniently the case on earth. The ocean thermal sources carry heat and a large flux of minerals, as is also the case between continents and oceans. This helps to preserve a constant flux of chemical energy. There is also a constant energy input from the sun.

In a way, one can say that life is a parasite of all the available energy fluxes. Contrary to Inert Objects, Living Organisms survive only with the constant help of their environment and are far from being autonomous, and at equilibrium, as we love to think of ourselves. One point is that Inert Objects absorb energy and matter at their creation, and then remain static with essentially no interactions with the environment. On the contrary Living Beings need the input of matter and energy all the time. In this way, wanting to preserve life is a bet on the future, on the whole span of time.

\section{EATING IS MOVING}

A first understanding of why life invaded earth can be seen simply from this predatory need to survive. When a self-maintaining form appeared, it survived and thrived by eating (and changing) its environment. The life form could continue for a long time only if it were able in some way to reach out to another unperturbed place. The need for predation can be seen as the origin of movement. In other words, the self-maintenance of shapes encouraged a need for the active collecting of resources, hence the incursion into the world.

The basic reasoning that is deduced from considering the first organized selfmaintaining structure can be easily applied to human activities. For instance the strategy of seeking outside ones traditional boundaries, when the environment is exhausted, is still a part of the nomadic tradition. When the environment is used up, Nomads move on, returning after the environment reverts to its original state. This works well for small populations. For higher densities, there is a requirement for either the expansion to new territories, or the invention of new regulations, that organizes the fluxes, thus creating a new shape that maintains itself at a higher level, as in cities and societies.

This need for motion in order to fulfil the requirement of food for permanent maintenance is also seen clearly in the birth of multicellularism, as in Dictyolstelium Discoideum. The amibia live their lives happily enough separately as long as there is enough food and humidity but group together when there is stress. The first consequence of grouping is to form a slug that moves away seeking a better place. Grouping to form a slug has an evident benefit: the slug is moving much faster and efficiently than the separate amibia. Another Myxomycete, Physarum Polycephalum, is also interesting: it groups the 
cells into a multinuclear macroscopic cell, which is then able to perform large cyclical contractions, like a muscle, just behind its periphery. The effect of these contractions is to pump the rest of the cellular material and push it to a new front, thus helping, in an efficient way, the whole group to advance.
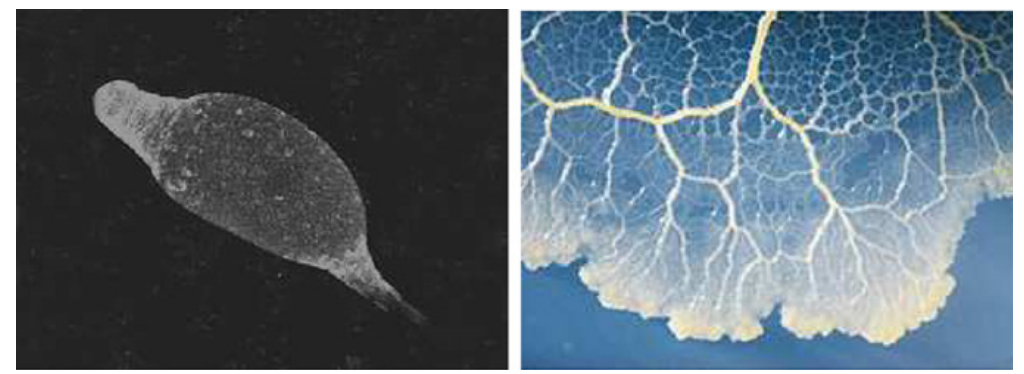

Figure 8. A moving slug of Dictyolstelium Discoideum, composed of tens of thousands of previously separated amebia (left), and a detail of a Physarum Polycephales mould, with and new advancing front followed by vessels conducting the previous material into the new front.

Plants seems to be an example of the stationary, compared to moving animals. Yet, plants are constantly moving, only at a much smaller pace, expanding continuously their roots and branches to reach for more absorption. They, too, seek outside into their environment to re-establish or recreate themselves, preserving their local shape and organization.

The first living organisms may not have possessed their own means of motion that required a particular internal dynamic structure but simply used the motion from the surrounding physical world, such as fluid currents, to transport nutrients to them or to be transported by them. They just had to keep the possibility of surviving while being transported in potentially inadequate environments. However, the perception of the state of the environment and the tendency to move toward an unperturbed environment (chemotaxism) can be seen as a basic consequence of permanent maintenance. This ability to explore the environment and to react to it necessarily means a higher degree of structure, internal shape, and higher needs.

\section{NEED IS INSTABILITY}

The higher need for more complex and able structures is the source of a fundamental instability. If a living shape does not have the needed input to maintain itself, it dies. But its disappearance can take time. During the decay some protection mechanisms can be put in place, over time. One, would be to be able to change shape to seek nutrients more easily outside. But this change of shape induces different needs. In particular, motion and seeking, require a more complex and changing shape, reacting to the environment. Paradoxically, all those more complex structure and changes can be achieved only with a higher input. In other words, becoming more complex to ensure the need requirements induce more needs, leading to a dangerous instability. 
This instability can be seen as the fundamental origin of not only the expansion of life but the increase of complexity. Complexity is the outcome of fulfilling ever increasing needs. Instability could explain the exponential growth we can see everywhere, from the size of the human populations and their energy intake, to the global extension of life forms and the variety of shapes.

Instabilities are dangerous as their exponential growth are explosions. In general, an instability is limited by a finite amount of energy. It can lead to stabilization, in the best cases, when the rate of growth is not too large and the energy can be renewed quickly enough. When the energy is consumed and not renewed, it leads to a subsequent collapse. Such explosions followed by a disappearance are commonly observed now with the green algae booms feeding on the artificial agriculture nutriments. It can also be seen in the geological time span as periods of life form booms followed by massive disappearance.
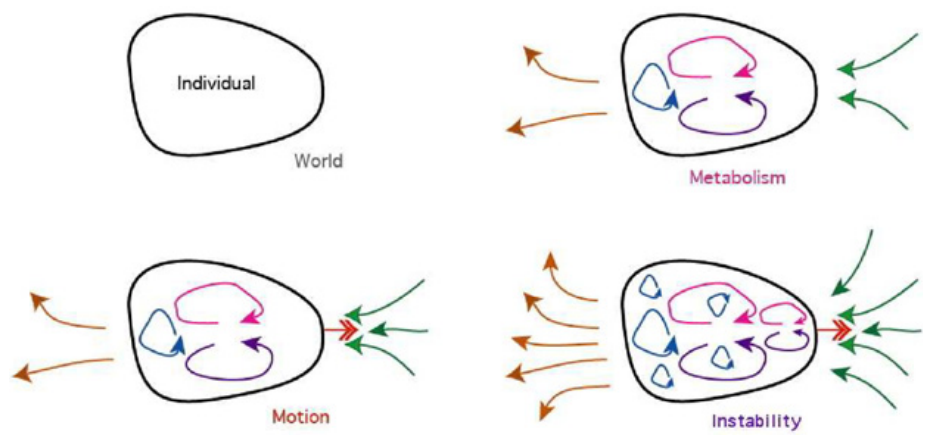

Figure 9. If an individual is considered (separated from the world), it can be either static (left), or alive (right), and then absorbing and rejecting permanently to maintain itself, through metabolism. Changes in the environment induce active seeking, i.e. motion, which in turn induce higher needs (and rejections).

\section{SEEKING IS WAR ...AND ITS OPPOSITE}

When we consider the effects of constant need induced by constant regeneration, the way to take the energy and matter is synonymous with predation, attacks, and waging war on the outside to capture the needs. It is the major origin of wars, raids and ransacks. The stabilization of this income to secure their sources and directed fluxes, is also at the origin of wars.

In general competition and the idea of wars have pervaded the whole way of thinking. It is also the way we think about illness. In disease we are essentially attacked by other living forms, which feed upon our bodies. To combat disease we believe our only recourse is to think about fighting back, with other war means.

However, the increased needs and the securing of them have induced more complex structures, able to react to ever more varied situations. This can be done only through the cooperation of many different parts. Similarly, the same need for the stabilization of constant fluxes has induced at larger scales the appearance of global cooperating interactions, groups, societies, specializations all linked to the presence of a spatial 
structure (nest, cities, palaces, suburbs, not to speak of pluricellular organisms, with conduits organizing circulations to the various organs.

This way of becoming more complex and organized can this be seen as another consequence of having to secure needs. It can be seen as the opposite of the instability seeking war outside. Rather, it is grouping together and collaborating for stabilization. In this way increasing needs have paradoxical consequences.

Many relationships could be seen that way, as parasite and host. Parasites are seen negatively and need to be destroyed. Whereas, when a good recognition of both the needs of the parasite and the host is achieved a harmonious a symbiotic relationship can then be seen as positive. This symbiotic relationship is a way of securing the needs to maintain both shapes. This maintenance is based on the collaboration of two living organisms for a priori an infinite length of time, while war and destruction is only a momentary solution. This benefit in time could explain the expansion of collaboration and ecosystems.

\section{PRACTICAL APPLICATION}

These interactions can be examined at all level of organization, from inside the cell, to between the cells, to between the individuals, to between the societies Changing the way of looking at interactions, first as shapes, and then at how to achieve the maintenance of these shapes is a paradigm shift that could have many practical applications.

Take for instance the case of illness and, in particular, cancer. Inside the human body, cancerous cells are known to change shape before invading the body. Instead of stating that the change of cell shape and mobility are a consequence of becoming cancerous, originating for instance from unexplained mutations, one could ask if this change of shape and mobility, is not simply a signal of necessary requirements not been met for the normal preservation of the cell. This unmet requirement necessitates the seeking out of a higher income (instability). This higher need occurring from stress explains directly the higher metabolisms for which cancer cells are known. It also explains their tendency to invade the rest of the body, seeking, and absorbing for their own advantages all the available resources.

Can this sudden change in shape and dynamics (behaviour) be rather seen as an indication that these cells are under stress and are looking for ways to opt out of their current environment? These cells were participating in a symbiotic relationship. When it was disrupted, they just opt for the other option for survival, the war side, becoming selfish again and seeking their need at the outside expense.

The change of the shape and behaviour of these cells is thought of as a sudden change in their status, from being normal internal cells composing of us, to abnormal, renegade cells now behaving like outside intruders, that have to be fought with war weapons. Up to now the only recipe is to try remove and destroy them. This is difficult as there is not much difference between the normal and abnormal cells. For the moment the best cure is chemotherapy, with is a strong poison, killing every living cell. It is administered at a limited dose so that proportionally more of the cells with a higher metabolism (among which are the cancer cells) are killed.

But is it the only way, or even the best way, to attempt to destroy cells which are just trying to survive? Couldnt we try to recognise their change in behaviour as unmet needs, and find a collaborative way to fulfill them, in order to re-establish the previous symbiotic 

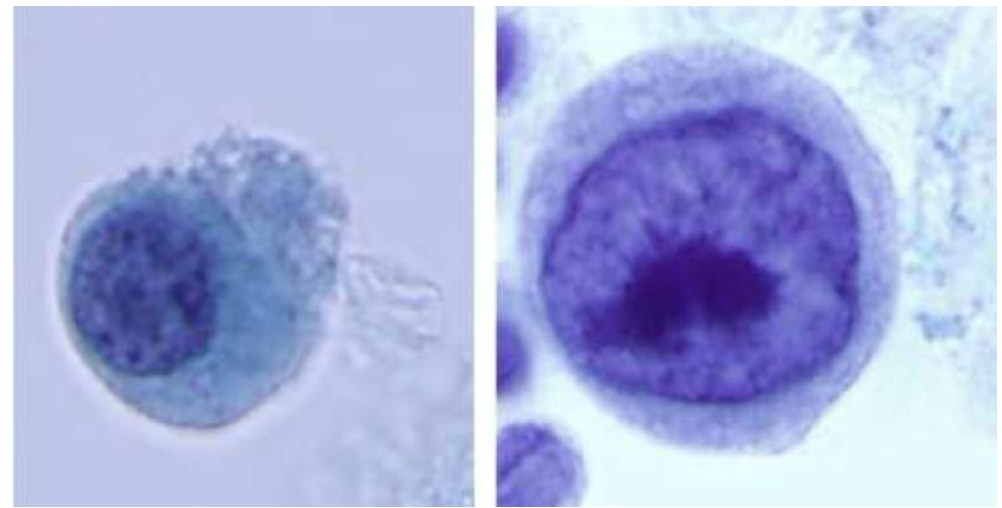

Figure 10. Normal (left) and Cancerous (right) Mesothelium cells. Shapes are the basic tools used to recognise normal cells from cancerous cells. Normal mesothelium cells have a small nucleus and a large cytoplasm; cancerous cells have a larger nucleus, pushing the cytoplasm at the periphery, with a concentrated nucleosome (chromatin, at the center). In this way most of the genome is spread around, dilated, accessible and readable. This resembles a stress mode where all the information and possible recipes to survive are tried at the same time.

relationship? In this way it would be to look at the consequence of maintaining shape not by waging war but through cooperation.

This change and meeting of requirements could be the only reasonable explanation for the few spontaneous cure of cancers, or even the fact that cancers are also, like most of the diseases, sensitive to the placebo effect.

\section{PUTTING THE SHAPE FIRST AS A WAY TO SEE THINGS DIFFERENTLY}

The study of organic shapes should be reconsidered, not only as the trace of a prehistoric state of Biology, a compilation of the diversity of shapes, but as a study of key ingredient in the dynamics of living organisms. Certain mysteries of life may reveal themselves under a new perspective of putting shape and its maintenance at the origin of life. Some essential consequences of this basic fact have been outlined here.

We can see that shapes are fundamental, from the reason why life invaded earth and colonised it, and complex, from the tendency to destroy their surroundings when invading.

The main interest of studying shapes is to look at the origin of life from a new perspective, to turn back the tables. Putting shape and its maintenance in life before the genetic code, could be a way to see challenges differently, with the outcome of trying to solve them in new and different ways. Cancer is one mystery that could be solved by looking at shapes.

Cooperation and collaboration is a long way from invasion and control. The way we approach everything in life is based upon a primitive warlike approach to survival. Whereas, long term development or more complex and able structures is achieved only through cooperation. 
A paradigm shift would necessarily coach society away from the killer paradigm to develop an alternate understanding of why harmony is disrupted and to explore ways to restore harmony outside the traditional methods.

Everything seems to be interconnected and to work in similar ways. Seeing shape as the beginning of a process is another step toward understanding the evolutionary paradigm from simple survival to complexity and wars to cooperation and harmony.

\section{References}

Kupiec, J.-J., Sonigo, P. 2000. Ni Dieu ni Gène. Points Seuil.

Hersen, P., 2004. On the Crescentic Shape of Barchan Dune. Eur. Phys. Jour. B.

Chaisson, E.J., 2001. Cosmic Evolution: The Rise of Complexity in Nature. Harvard University Press, Cambridge. 